E3S Web of Conferences 1, 05006 (2013)

DOI: $10.1051 / \mathrm{e} 3$ sconf/20130105006

(C) Owned by the authors, published by EDP Sciences, 2013

\title{
Method Development for the Determination of Fluorine in Water Samples via Molecular Absorption of CaF Using A High-Resolution Continuum Source Electrothermal Atomic Absorption Spectrophotometer
}

\author{
N. Ozbek ${ }^{1}$ and S. Akman ${ }^{2}$ \\ ${ }^{1}$ Istanbul Technical University, Faculty of Arts and Sciences, Department of Chemistry, 34469 Maslak, Istanbul, Turkey, \\ nil.ozbek@itu.edu.tr \\ ${ }^{2}$ Istanbul Technical University, Faculty of Arts and Sciences, Department of Chemistry,34469 Maslak, Istanbul, Turkey, \\ akmans@itu.edu.tr
}

\begin{abstract}
In this study, determination of fluorine in water samples was established via the rotational fine structured molecular absorption of calcium mono-fluoride $(\mathrm{CaF})$ at $606.440 \mathrm{~nm}$ using a high-resolution continuum source electrothermal atomic absorption spectrophotometer. To generate $\mathrm{CaF}$ in the gas phase of graphite furnace, samples were pipetted together with calcium as its nitrate form. The effect of amount of calcium, graphite furnace program, coating the graphite tube and platform with $\mathrm{Zr}$ and $\mathrm{Ir}$ and the use of modifier $(\mathrm{Pd}, \mathrm{Mg}$ and $\mathrm{Pd}+\mathrm{Mg})$ on the sensitivity and linearity were investigated. In the presence of $40 \mu \mathrm{g}$ of $\mathrm{Ca}$, a perfect linearity up to $0.1 \mu \mathrm{g}$ of $\mathrm{F}$ was maintained applying a pyrolysis temperature of $700^{\circ} \mathrm{C}$ and a molecule forming temperature of $2250^{\circ} \mathrm{C}$ in pyrolytically coated platforms. The limit of detection and characteristic mass of the method were $0.21 \mathrm{ng}$ and $0.13 \mathrm{ng}$ of $\mathrm{F}$, respectively.
\end{abstract}

Key words: High-resolution continuum source electrothermal atomic absorption spectrometry (HR-CSETAAS); Molecular absorption spectrometry (MAS); Fluorine determination; Calcium monofluoride (CaF); Water samples

\section{Introduction}

Fluorine is an essential element trace element for human health, but exposure of fluorine in excess amounts may cause dental and/or skeletal fluorosis (Wang et al., 2004). Even though absence of fluorine does not cause dental caries, ingestion of fluorine may help to reduce tooth decay. The effect of fluorine depends on its total daily intake from all sources. Drinking water is typically the largest source for fluorine; other methods of fluorine therapy include fluoridation of toothpaste, salt, and milk (Petersen et al., 2004). Since the amount of fluorine intake is important, fluorine contents of water, beverages and many food and drink samples have been investigated deeply in literature, which were well reviewed by Huang et al.(2006).

Atomic absorption spectrometry (AAS) is a traditional analytical method for quantitative determination of trace metals and metalloids. The traditional line source atomic absorption spectrometry cannot be used for determination of fluorine because the main resonance line of $\mathrm{F}$ is located at $95 \mathrm{~nm}$ which cannot be instrumentally applied. With the new generation high resolution continuum source atomic absorption spectrometer (HR-CS-AAS), it is possible to determine fluorine with any suitable diatomic molecule formed with a selected metal at its any appropriate rotational hyperfine rotational absorption wavelength. There are limited number of studies on the determination of fluorine by HR-CS-AAS using molecular absorption by several diatomic molecules like AlF (Ozbek and Akman, 2012), GaF (Gleisner et al., 2010), CaF (Mores et al., 2011) and SrF (Ozbek and Akman, 2012).

This study describes a new method for determination of fluorine in water matrix by HR-CSETAAS using molecular absorption line of $\mathrm{CaF}$. The experimental parameters were optimized and method was validated.

\section{Materials and Methods}

A graphite furnace atomic absorption spectrophotometer Analytik Jena ContrAA 700 highresolution continuum source atomic absorption 
spectrophotometer equipped with MPE auto sampler (Analytik Jena, Jena, Germany) and a $300 \mathrm{~W}$ xenon shortarc lamp (XBO 301, GLE, Berlin, Germany) operating in a hot-spot mode as a continuum radiation source was used for all measurements. The equipment presents a compact high-resolution double echelle monochromator and a charge-coupled device (CCD) array detector with a resolution of about $5 \mathrm{pm}$ per pixel. Measurements were carried out at $606.440 \mathrm{~nm}$ molecular absorption line of $\mathrm{CaF}$ with 3 pixels (central pixel \pm 1 ). All measurements were performed using pyrolytically coated graphite tubes with integrated PIN platform (Analytik Jena Part No. 407-A81.025).

In all dilutions a high-purity water (resistivity 18.2 $\mathrm{M} \Omega . \mathrm{cm}$ ) obtained by a TKA reverse osmosis and a TKA deionizer system (TKA Wasseraufbereitungsysteme $\mathrm{GmbH}$, Niederelbert Germany) was used. Inorganic acids and reagents were of analytical grade $\left(\mathrm{HNO}_{3}, 65 \%(\mathrm{v} / \mathrm{v})\right.$, Merck, Darmstad, Germany). The fluorine standard was prepared from sodium fluoride (Merck, Darmstad, Germany). A stock solution of $10000 \mathrm{mg} \mathrm{L}^{-1}$ calcium as molecule forming agent was prepared from barium nitrate (Merck, Darmstadt, Germany). The waste water standard reference material SPS-NUTR-WW2 was provided from LGC Standards (Middlesex, England). Drinking and tap water samples had obtained from different cities of Turkey.

Determination of fluorine was performed using the rotational molecular absorption line of diatomic $\mathrm{CaF}$ at $606.440 \mathrm{~nm}$ by HR-CS-AAS. Samples (or standard solutions) and $4000 \mu \mathrm{g} \mathrm{L}^{-1}$ of calcium as the nitrate were pipetted as $10 \mu \mathrm{L}$.

\section{Results and Discussion}

\section{Choice of molecule-forming reagent}

Since the method is based on the measurement of the molecular absorption of a diatomic molecule formed between the analyte with a selected element at one of its finely structured rotational lines, it is very important to choose the appropriate molecule. Diatomic molecule of $\mathrm{F}$ formed should be stable in gas phase. The diatomic species with bond dissociation energies above $500 \mathrm{~kJ}$ $\mathrm{mol}^{-1}$ are suitable for this purpose. Moreover, the molecular absorption sensitivity of the working wavelength for the diatomic molecule should be high enough to detect small $\mathrm{F}$ concentrations. It was expected that calcium would be an appropriate element for this purpose as well.

The 3D (i.e., time and wavelength-resolved) absorption spectra obtained for water sample spiked with $10 \mu \mathrm{g} \mathrm{L}^{-1} \mathrm{~F}$ in the vicinity of the molecular absorption peak of $\mathrm{CaF}$ at $606.440 \mathrm{~nm}$ is depicted in Figure 1. The background is low, and the MAS of CaF is smooth. There is no spectral overlap from the matrix. Therefore, all determinations of $\mathrm{F}$ throughout this study were performed using the rotational molecular absorption line of $\mathrm{CaF}$ at $606.440 \mathrm{~nm}$.

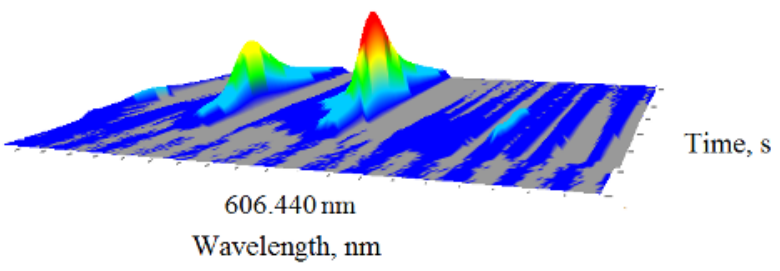

Fig. 1. Time and wavelength resolved absorption (3D) spectra obtained for water sample spiked with $10 \mu \mathrm{g} \mathrm{L}^{-1}$ in the vicinity of $606.440 \mathrm{~nm}$ molecular absorption peak of CaF. Ca: $4 \mathrm{mg} \mathrm{mL}^{-1}$.

\section{Optimization of graphite furnace program}

The graphite furnace program used for determination of $\mathrm{F}$ from the MAS of $\mathrm{CaF}$ at $606.440 \mathrm{~nm}$ should be designed to combine $\mathrm{Ca}$ and $\mathrm{F}$ while removing certain matrix consitituents that may cause interferences and forming $\mathrm{CaF}$. The maximum sensitivity is obtained with $700^{\circ} \mathrm{C}$ pyrolysis temperature with a molecule forming temperature $2250^{\circ} \mathrm{C}$.

\section{Use of modifiers}

In this study, the effects of $\mathrm{Pd}, \mathrm{Pd}+\mathrm{Mg}, \mathrm{Ir}$ and $\mathrm{Zr}$ modifiers on the sensitivities and pyrolysis and atomization temperatures were investigated. When Pd or $\mathrm{Pd}+\mathrm{Mg}$ modifiers were used in the graphite furnace, no significant improvement was observed. In contrast, the sensitivities were reduced.

\section{Optimization of the amount of calcium}

The amount of calcium should be high enough to produce constant and maximum $\mathrm{CaF}$ sensitivity for all $\mathrm{F}$ concentrations in the matrix of the sample. In this case, $\mathrm{CaF}$ concentration and its absorbance would linearly be proportional with $\mathrm{F}$ concentration. Otherwise, non-linear relationship between $\mathrm{F}$ and $\mathrm{CaF}$ concentrations occurs which is the case in some applications. The absorbances for $\mathrm{CaF}$ in water samples linearly increased up to $20 \mu \mathrm{g}$ $\left(10 \mu \mathrm{L}\right.$ of $\left.2000 \mathrm{mg} \mathrm{L}^{-1}\right)$ of $\mathrm{Ca}$ and remained almost constant at higher $\mathrm{Ca}$ concentrations. In this study, being on the safety side, $40 \mu \mathrm{g}$ of Ca was used as the molecule forming element for all determinations.

\section{Linearity}

The sensitivities and linearities of working curves for $\mathrm{F}$ obtained from the MAS of $\mathrm{CaF}$ at $606.440 \mathrm{~nm}$ were investigated in the presence of $40 \mu \mathrm{g}$ of $\mathrm{Ca}$. The perfect linearity was obtained up to $10 \mathrm{mg} \mathrm{L}^{-1}$ of $\mathrm{F}$ which was expected to be much higher than $\mathrm{F}$ concentrations in samples. On the other hand, in complex matrices, $\mathrm{Ca}$ and $\mathrm{F}$ interact not only with each other but also with the other constituents. In this case, concentration of $\mathrm{CaF}$ formed will not be the same with that in standard solutions. In this case, to eliminate the non-spectral interferences, standard addition technique seems to be more appropriate 
Table 1. Fluorine concentrations ${ }^{1}$ in drinking water, tap water and certified reference samples found by linear calibration and standard addition techniques via molecular absorption of $\mathrm{CaF}$ at $606.404 \mathrm{~nm}$. Ca: $4 \mathrm{mg} \mathrm{mL}^{-1} ; \mathrm{N}=3$.

\begin{tabular}{lccc}
\hline \multicolumn{1}{c}{ Sample } & $\begin{array}{c}\text { Expected Value } \\
\left(\mu \mathrm{g} \mathrm{mL}^{-1}\right)\end{array}$ & $\begin{array}{c}\text { Value Found by Linear } \\
\text { Calibration } \\
\left(\mu \mathrm{gL} \mathrm{mL}^{-1}\right)\end{array}$ & $\begin{array}{c}\text { Value Found by Standard } \\
\text { Addition } \\
\left(\mu \mathrm{mL}^{-1}\right)\end{array}$ \\
\hline Waste water (SPS-NUTR WW2) & $10.00 \pm 0.10$ & $9.80 \pm 0.06$ & $9.70 \pm 0.06$ \\
Bottled Drinking Water 1 & 0.01 & $<\mathrm{LOD}$ & $<\mathrm{LOD}$ \\
Bottled Drinking Water 2 & 0.25 & $0.23 \pm 0.01$ & $0.24 \pm 0.05$ \\
Tap Water Sample 1 & $\mathrm{NG}^{3}$ & $0.59 \pm 0.06$ & $0.60 \pm 0.03$ \\
Tap Water Sample 2 & $\mathrm{NG}^{3}$ & $0.87 \pm 0.04$ & $0.85 \pm 0.04$ \\
Tap Water Sample 3 & $\mathrm{NG}^{3}$ & $0.41 \pm 0.03$ & $0.40 \pm 0.08$ \\
Tap Water Sample 4 & $\mathrm{NG}^{3}$ & $0.28 \pm 0.02$ & $0.25 \pm 0.06$ \\
Tap Water Sample 5 & $\mathrm{NG}^{3}$ & $0.75 \pm 0.06$ & $0.77 \pm 0.03$ \\
Tap Water Sample 6 & $\mathrm{NG}^{3}$ & $0.63 \pm 0.04$ & $0.65 \pm 0.04$ \\
\hline
\end{tabular}

${ }^{1}$ Results presented are mean \pm standard deviation $(\mathrm{N}=3)$.

${ }^{2}$ The fluoride concentrations given on the bottle of water sample or certified value of CRM

${ }^{3} \mathrm{NG}$ : not given

technique for quantifications of $\mathrm{F}$ in real samples. However, the results in real water samples found by linear calibration and standard addition techniques were not significantly different. This shows that the abovementioned problems (non-spectral interferences) did not occur in the determination of $\mathrm{F}$ in water samples which can be attributed to the simple matrices of the samples.

\section{Figures of merit}

In general, limit of detection (LOD) is calculated according to 3 times the standard deviation $(\sigma)$ for 10 repetitive pipettings of the blank to the furnace $(3 \sigma /$ slope of calibration graph) whereas the characteristic mass is calculated as the concentration of $\mathrm{F}$ corresponding to 0.0044 A i.e. $0.0044 /$ slope of calibration graph. However, the standard deviations as well as the sensitivities may show some small discrepancies from day to day. In addition, the sensitivity and thus LOD and characteristic mass values depend on the amount of $\mathrm{Ca}$ as well. In this study, in order to determine LOD, 3 times the standard deviation for 10 repetitive pipettings of $10 \mu \mathrm{L}$ of 4000 $\mathrm{mg} \mathrm{L}^{-1}$ of $\mathrm{Ca}$ with a water sample containing one of the lowest analyte content. The average LOD and the characteristic mass values found on various days were $0.21 \mathrm{ng}$ and $0.13 \mathrm{ng}$ (or $0.021 \mu \mathrm{g} \mathrm{mL}^{-1}$ and $0.013 \mu \mathrm{g} \mathrm{mL}^{-1}$ for $10 \mu \mathrm{L}$ of pipetting), respectively. The accuracy of the method was tested using a certified reference waste water sample (SPS-NUTR-WW2). The results were in good agreement with certified values as well as the precision was satisfactory $(\mathrm{RSD}<15 \%)$. Finally, $\mathrm{F}$ contents of several water samples were determined linear calibration technique and standard addition technique.

\section{Conclusion}

A novel method is proposed for the determination of fluorine in different water samples using HR-CS-ET AAS via the MAS of calcium monofluoride formed in gas phase. In this method, there was no need to use modifiers or to cover the tubes and platform with $\mathrm{Zr}$ or Ir. Validation of method was performed with wastewater $\mathrm{CRM}$ and fluorine concentrations could be determined in the uncertainty limits of the certified value. The results in CRM and real samples obtained by linear calibration technique and standard addition were nearly similar but to be on safe side, it is advised to apply the standard addition technique for any quantification.

\section{References}

Gleisner H, Welz B, Einax JW, Optimization of fluorine determination via molecular absorption of gallium mono-fluoride in a graphite furnace using a highresolution continuum source spectrometer, Spectrochim. Acta Part B, 2010, 65:864-869.

Huang M D, Becker-Ross H, Florek S, Heitmann U, Okruss M, Determination of halogens via molecules in the air acetylene flame using high-resulution continuum source absorption spectrometry Part 1: Fluorine, Spectrochim. Acta B, 2006, 61:572-578.

Ozbek N, Akman S, Method development for the determination of fluorine in toothpaste via molecular absorption of aluminum mono fluoride using a high- resolution continuum source nitrous oxide/acetylene flame atomic absorption spectrophotometer, Talanta, doi:10.1016/j.talanta. 2012.03.034. 
Ozbek N, Akman S, Method development for the determination of fluorine in water samples via the molecular absorption of strontium monofluoride formed in an electrothermal atomizer, Spectrochim. Acta Part B, 2012, 69:32-37.

Petersen PE, Lennon MA. Effective use of fluorides for the prevention of dental caries in the 21 st century: the WHO approach, Community Dent Oral Epidemiol. 2004, 32(5):319-21
S. Morés, G. C. Monteiro, F. S. Santos, E. Carasek, B. Welz, Determination of fluorine in tea using highresolution molecular absorption spectrometry with electrothermal vaporization of the calcium monofluoride CaF, Talanta, 2011, 85:2681-2685.

Wang B, Zheng B, Zhai C, Yu G, Liu X, Relationship between fluorine in drinking water and dental health of residents in some large cities in China, Environ. Int. 2004, 30:1067-1073. 